\title{
Asynchronous Time Encoding: An Approach to Sub-Nyquist Rate Sampling
}

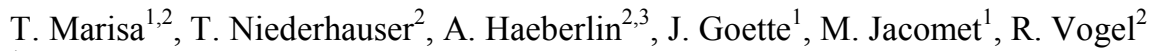 \\ ${ }^{1}$ HuCE-Microlab, Bern University of Applied Sciences, Biel, Switzerland, thanks.marisa@bfh.ch \\ ${ }^{2}$ ARTORG Cardiovascular Engineering, University of Bern, Bern, Switzerland \\ ${ }^{3}$ Department of Cardiology, Bern University Hospital, Bern, Switzerland
}

\section{Introduction}

Continuous long-term implantable signal acquisition devices have high energy and memory requirements, in order to capture and store data for a long period. The memory requirement could be reduced by compressing the data, but the computations to compress the data by existing methods result in a substantial increase in the energy consumption. We present as a solution an adaptive time-encoder that captures signal transition-times at a rate depending on the activity of the signal, as opposed to either constant Nyquist-rate or asynchronous and adaptive signal amplitude sampling [1,2].

\section{Operation Principle}

We constructed a mixed-signal circuit simulation model. The principle of operation is to capture and encode the changes in time, $\Delta \mathrm{t}$, it takes for the signal to make a predefined amplitude change $\Delta \mathrm{V}$. The signal type, $\Delta \mathrm{V}$, and timer resolution controls the accuracy with which we can reconstruct our signal, and the amount of data to be stored.

\section{Results}

We carried out simulations with esophageal and surface ECG signals collected from our clinical trials. We also developed a custom reconstruction algorithm for our irregularly sampled data. The performance results of the encoder are presented in Table 1:

\begin{tabular}{|c|c|}
\hline Parameter & Value \\
\hline Digital supply voltage & $0.8 \mathrm{~V}$ \\
\hline Analog Supply voltage & $1.2 \mathrm{~V}$ \\
\hline Average signal compression & 4 times \\
\hline Average ADC power consumption & $7 \mu \mathrm{W}$ \\
\hline$\Delta$ V resolution & $4-8 \mathrm{bits} / \mathrm{mV}$ \\
\hline Equivalent ADC Resolution & $6-12 \mathrm{bits}$ \\
\hline
\end{tabular}

Table 1: Time encoder parameters.

The time encoder is able to operate at power levels as low as $7 \mu \mathrm{W}$ whilst providing acceptable signal quality for medical diagnosis.

\section{Conclusion}

The proposed time encoder offers a flexible architecture that offers signal activity dependent data acquisition and can be customized to suit a particular signal acquisition scenario. These combined attributes minimize the system's energytimes-memory product. The ADC achieves an average compression of 4 times on esophageal and surface ECGs compared to the classical Nyquist ADC architectures and needs no computations for this compressed data acquisition. We thus reduce both memory and energy requirements and in turn the volume of the implantable signal capturing system.

\section{References}

[1] H.V. Sorensen Sayiner and T.R. Viswanathan. A level-crossing sampling scheme for A/D conversion. IEEE Transactions on Circuits and Systems II, 43:335-339, 1996.

[2] Yannis P. Tsividis. Event-Driven Data Acquisition and Digital Signal Processing - A Tutorial. IEEE Trans. on Circuits and Systems, 57-II (8):577-581, 2010. 